\title{
Identification of Multipotent Stem Cells in Human Brain Tissue Following Stroke
}

\author{
Kotaro Tatebayashi,, ${ }^{1, *}$ Yasue Tanaka, ${ }^{1,2, *}$ Akiko Nakano-Doi,," Rika Sakuma, \\ Saeko Kamachi, Manabu Shirakawa, Kazutaka Uchida, Hiroto Kageyama, \\ Toshinori Takagi, Shinichi Yoshimura, ${ }^{1}$ Tomohiro Matsuyama, ${ }^{2}$ and Takayuki Nakagomi ${ }^{2}$
}

Perivascular regions of the brain harbor multipotent stem cells. We previously demonstrated that brain pericytes near blood vessels also develop multipotency following experimental ischemia in mice and these ischemiainduced multipotent stem cells (iSCs) can contribute to neurogenesis. However, it is essential to understand the traits of iSCs in the poststroke human brain for possible applications in stem cell-based therapies for stroke patients. In this study, we report for the first time that iSCs can be isolated from the poststroke human brain. Putative iSCs were derived from poststroke brain tissue obtained from elderly stroke patients requiring decompressive craniectomy and partial lobectomy for diffuse cerebral infarction. Immunohistochemistry showed that these iSCs were localized near blood vessels within poststroke areas containing apoptotic/necrotic neurons and expressed both the stem cell marker nestin and several pericytic markers. Isolated iSCs expressed these same markers and demonstrated high proliferative potential without loss of stemness. Furthermore, isolated iSCs expressed other stem cell markers, such as Sox2, $c-m y c$, and Klf4, and differentiated into multiple cells in vitro, including neurons. These results show that iSCs, which are likely brain pericyte derivatives, are present within the poststroke human brain. This study suggests that iSCs can contribute to neural repair in patients with stroke.

Keywords: ischemia, stroke, multipotent stem cells, human, pericyte

\section{Introduction}

I SCHEMIC STROKE OFTEN results in severe and irreversIible central nervous system (CNS) dysfunction. While increasing numbers of stroke patients now benefit from treatments such as neuroendovascular intervention and thrombolytic therapy $[1,2]$, many still suffer from permanent sequela due to the limited time window for these therapies and frequent unsuccessful recanalization.

Stem cell-based therapies have emerged as a promising alternative therapeutic option for patients with ischemic stroke. Among several types of stem cells, neural stem/progenitor cells (NSPCs) are a strong therapeutic candidate because they can differentiate into various neural cell types, including neurons. Accumulating evidence indicates that NSPCs are present not only in the rodent [3-7] but also in the human CNS [8-10]. Previous rodent experiments have also demonstrated that many cell types in the healthy mature brain, such as astrocytes in the subventricular zone (SVZ) $[3,4]$, ependymal cells [7], oligodendrocyte precursor cells [5], and resident glia [11], are potential sources of NSPCs.

Although the precise origin of NSPCs under pathological conditions remains unclear, using a mouse model of cerebral infarction, we previously reported that brain pericytes near blood vessels developed multipotent stem cell activity in response to ischemia/hypoxia and differentiated into various neural lineages $[12,13]$. These results indicate that ischemiainduced multipotent stem cells (iSCs) originating from pericytes (iPCs) function as NSPCs in mice and may contribute to neural regeneration [12-14].

However, it remains unclear if pericytes can also transition to multipotency in human brain following stroke. Moreover, it is essential to understand the regulation of human iSC proliferation and differentiation for possible applications in stem cell-based stroke therapy. Although our previous study identified nestin ${ }^{+}$cells presumably containing iSC populations in poststroke human autopsied brains [15], there is still no direct evidence that

\footnotetext{
${ }^{1}$ Department of Neurosurgery, ${ }^{2}$ Institute for Advanced Medical Sciences, Hyogo College of Medicine, Nishinomiya, Japan.

*These authors contributed equally to this work.
}

(C) Kotaro Tatebayashi et al., 2017; Published by Mary Ann Liebert Inc. This article is available under the Creative Commons License CCBY-NC (http://creativecommons.org/licenses/by-nc/4.0). This license permits non-commercial use, distribution and reproduction in any medium, provided the original work is properly cited. Permission only needs to be obtained for commercial use and can be done via RightsLink. 
iSCs are present or induced within the poststroke human brain.

In this study, using brain samples obtained from stroke patients who needed partial lobectomy as well as decompressive craniectomy for diffuse cerebral infarction, we investigated whether iSCs can be isolated from the poststroke human brain. We then examined the localization, gene expression patterns, proliferative potential, and multipotency of human iSCs.

\section{Materials and Methods}

\section{Patients}

The study protocol was reviewed and approved by the Ethics Committee of Hyogo College of Medicine (approval number: 1776). Brain samples were obtained from the poststroke areas in two patients who satisfied the following criteria: (1) requiring both decompressive craniectomy and partial lobectomy for diffuse cerebral infarction, (2) $>20$ years of age, and (3) willing to provide written informed consent. The diagnosis of cerebral infarction was made by neurosurgeons and radiologists according to physical examination and neuroimaging [eg, brain computed tomography (CT), magnetic resonance imaging (MRI), and magnetic resonance angiography (MRA)]. Due to ethical considerations, the neurosurgeons involved in diagnosis and surgical treatment of cerebral infarction were not involved in subsequent studies on the identification, isolation, and characterization of iSCs from excised tissue.

Patients with the following conditions/diseases were excluded from the study: (1) presence of a malignant tumor, (2) presence and history of major infectious diseases (infectious disease category $1-5$, infection designated by government ordinance, new strains of influenza, and new infectious disease), and (3) patients judged as unsuitable by the attending doctor.

\section{Histological examination and terminal dUTP nick-end labeling of poststroke human brain tissue sections}

For histochemical analysis, poststroke brain tissue samples were fixed overnight with periodate-lysine-paraformaldehyde (PLP), cryoprotected in $30 \%$ sucrose, frozen at $-80^{\circ} \mathrm{C}$, and cut into $20-\mu \mathrm{m}$ sections using a cryostat. Sections were then stained with hematoxylin and eosin $(\mathrm{H} \& \mathrm{E})$, processed for immunohistochemistry, or subjected to terminal dUTP nickend labeling (TUNEL). TUNEL was performed using an in situ apoptosis detection kit according to the manufacturer's instructions (Takara Biomedicals, Kusatsu, Japan), followed by counterstaining with methyl green solution (Wako, Osaka, Japan) as described [16].

\section{Immunohistochemistry}

Brain sections were immunostained with antibodies against Tuj1 (Stemcell Technologies, Vancouver, BC, Canada), microtubule-associated protein 2 (MAP2; Sigma, St. Louis, MO), glial fibrillary acidic protein (GFAP; Millipore, Temecula, CA), S-100 $\beta$ (Abcam, Cambridge, United Kingdom), nestin (Santa Cruz Biotechnology, Dallas, TX), von Willebrand factor (vWF; Santa Cruz Biotechnology), alpha smooth muscle actin ( $\alpha$ SMA; LifeSpan Biosciences, Seattle, WA), and neuron-glial antigen 2 (NG2; Millipore). Primary antibodies were visualized using Alexa Fluor 488- or 555conjugated secondary antibodies (Molecular Probes, Eugene, OR). Nuclei were counterstained with 4',6-diamino-2phenylindole (DAPI; Kirkegaard \& Perry Laboratories, Gaithersburg, MD). Images were captured using a confocal laser microscope (LSM780; Carl Zeiss, Jena, Germany).

\section{Cell culture and immunocytochemistry}

Human ischemic tissue was dissociated into single-cell suspensions by passage through 23- and 27-G needles. For adherent cultures, cells were seeded on $10-\mathrm{cm}$ dishes (Corning, NY) in Dulbecco's modified Eagle's medium/F12 (DMEM/F12; Invitrogen, Carlsbad, CA) containing basic fibroblast growth factor (bFGF; $20 \mathrm{ng} / \mathrm{mL}$; Peprotech, Rocky Hill, NJ), epidermal growth factor (EGF; $20 \mathrm{ng} / \mathrm{mL}$; Peprotech), $10 \%$ fetal bovine serum (FBS), and N2 (1\%; Invitrogen). When the cells reached confluence, they were deplated using trypsin, reseeded, and propagated under the same conditions.

Passage 5-10 cells (iSCs) were characterized by immunocytochemistry, reverse transcriptase-polymerase chain reaction (RT-PCR), and fluorescence-activated cell sorting (FACS). Immunocytochemistry was performed using antibodies against Ki67 (BD Pharmingen, San Jose, CA), nestin (Santa Cruz Biotechnology), $\alpha$ SMA (LifeSpan Biosciences), NG2 (Millipore), platelet-derived growth factor receptor beta (PDGFR $\beta$; Santa Cruz Biotechnology), and GFAP (DAKO, Glostrup, Denmark). Bound primary antibodies were visualized using Alexa Fluor 488- or 555conjugated secondary antibodies (Molecular Probes).

In another set of experiments, iSCs were incubated in a neural-conditioned medium that promoted the formation of neurosphere-like cell clusters [12-14,17,18]. In brief, iSCs in adherent culture were deplated with trypsin and incubated under floating culture conditions in a medium containing DMEM/F12, bFGF, EGF, 10\% FBS, and N2. On day 7 after seeding, cell clusters were picked out and subjected to RTPCR. On day 14, cell clusters were incubated for 7 days under conditions promoting neuronal differentiation with minor modifications [12-14,17,18]. The differentiated cells were then subjected to immunolabeling using an antibody against Tuj1 (Stemcell Technologies), followed by visualization using an Alexa Fluor 555-conjugated secondary antibody (Molecular Probes).

Putative iSCs were also cultured in osteogenic, adipogenic, or chondrocytic differentiation medium, respectively, according to the manufacturer's protocol (SC 006; R\&D systems, Minneapolis, MN) and then subjected to immunocytochemistry using antibodies against osteocalcin (R\&D Systems), fatty acid binding protein 4 (FABP4) (R\&D Systems), or aggrecan (R\&D Systems). In addition, iSCs incubated in an adipogenic differentiation medium were stained with the lipid-specific dye Oil Red O as described [19,20].

Human neural stem/progenitor cells (hNSPCs; Part. No. SCC003; Millipore), human brain-derived endothelial cells (ECs) (hECs; ACBRI-376; Cell Systems, Kirkland, WA), human brain-derived astrocytes (ACs) (hACs; \#1800; ScienCell Research Laboratories, Carlsbad, CA), and human bone marrow-derived mesenchymal stem cells (MSCs) 
(hMSCs; PT-2501, Lonza, Basil, Switzerland) were maintained in media specified by the supplier. Extracted mRNA samples were used as controls for RT-PCR analyses of iSC or iSC-derived cell expression patterns.

\section{Oxygen-glucose deprivation treatment}

In addition, iSCs were subjected to oxygen-glucose deprivation (OGD) treatment as described previously $[13,20]$. In brief, iSCs $\left(5 \times 10^{4}\right.$ cells/well) were plated on 12-well dishes (Iwaki, Tokyo, Japan) in a medium containing DMEM/F12, bFGF, EGF, $10 \%$ FBS, and N2. One day after plating, the medium was removed and replaced with glucose-free DMEM, FBS (2\%), bFGF, and $0.025 \mu \mathrm{g} / \mathrm{mL}$ recombinant human leukemia inhibitory factor (LIF; Millipore). The iSCs were then incubated under hypoxia $\left(1 \% \mathrm{O}_{2}\right)$ for 3 days using a hypoxia-inducing system (Bionix, SUGIYAMA-GEN, Tokyo, Japan). As a control, iSCs were incubated under normoxia for 3 days in glucose-containing DMEM (DMEM/ F12), FBS (2\%), bFGF, and $0.025 \mu \mathrm{g} / \mathrm{mL}$ LIF (Millipore).

\section{Cell proliferation}

For analysis of iSC proliferative capacity, $2 \times 10^{4}$ cells were plated in three wells of a 12-well plate and counted manually over 7 days. Doubling time was calculated using a formula described previously [21].

\section{Reverse transcriptase-polymerase chain reaction}

Total RNA was extracted from iSCs, hNSPCs, hECs, hACs, and hMSCs using an RNeasy Micro Kit (Qiagen, Hilden, Germany). cDNA was amplified according to the manufacturer's protocol as previously described $[12,13,17,20]$. The primer sequences used in this study are listed in Table 1.

\section{Flow cytometric analysis}

Suspended single iSCs or hMSCs were labeled with antibodies against CD19, CD44, CD45, CD90, CD105, and CD166 according to the manufacturer's instructions (R\&D Systems; SC017). Cell subpopulations were quantified using a fluorescence-activated cell sorter (FACS; BD LSRFortessa $^{\mathrm{TM}} \mathrm{X}-20$; BD Pharmingen), as described [18].

\section{Animal studies}

All experimental procedures were performed in accordance with a protocol approved by the Animal Care Committee of Hyogo College of Medicine and complied with the Guide for the Care and Use of Animals published by the Ministry of Education, Culture, Sports, Science and Technology in Japan. Six-week-old male CB-17/Icr-+/+Jcl mice (CB-17 mice; Clea Japan, Inc., Tokyo, Japan) were subjected to cerebral ischemia as described previously [12-14,17,18]. In brief, permanent focal cerebral ischemia was produced by ligation and interruption of the distal portion of the left middle cerebral artery (MCA). Under halothane inhalation, the left MCA was isolated, electrocauterized, and disconnected just distal to its crossing of the olfactory tract (the distal M1 portion). At $36 \mathrm{~h}$ after ischemic stroke, mice were anesthetized with sodium pentobarbital and perfused transcardially with $4 \%$ paraformaldehyde. The perfused brains were removed, cryoprotected in $30 \%$ sucrose, and sectioned on a cryostat. Then, coronal brain sections were prepared and subjected to H\&E staining and immunohistochemistry with antibodies against MAP2 (Sigma) and GFAP (DAKO, Glostrup, Denmark).

\section{Results \\ Clinical history of two patients with stroke undergoing decompressive lobectomy}

The first case (Case 1, Table 2) was a 79-year-old female admitted to our hospital complaining of consciousness disturbance and right conjugate deviation. On admission, she had left upper and lower limb hemiparesis and her National Institute of Health Stroke Scale score was 31. Brain CT

Table 1. Sequences of Human Primers Used for Reverse Transcriptase-Polymerase Chain Reaction

\begin{tabular}{|c|c|c|}
\hline Primers & Sequence $\left(5^{\prime} \rightarrow 3^{\prime}\right)(F:$ forward; $R:$ reverse $)$ & Size \\
\hline aSMA & F: GTGTTGCCCCTGAAGAGCAT; R: GCTGGGACATTGAAAGTCTCA & $109 \mathrm{bp}$ \\
\hline$\beta$-actin & F: GTCCTCTCCCAAGTCCACAC; R: GGGAGACCAAAAGCCTTCAT & $188 \mathrm{bp}$ \\
\hline CD31 & F: AACAGTGTTGACATGAAGAGCC; R: TGTAAAACAGCACGTCATCCTT & $148 \mathrm{bp}$ \\
\hline CDH5 & F: AAGCGTGAGTCGCAAGAATG; R: TCTCCAGGTTTTCGCCAGTG & $179 \mathrm{bp}$ \\
\hline c-myc & F: GCGTCCTGGGAAGGGAGATCCGGAGC; R: TTGAGGGGCATCGTCGCGGGAGGCTG & $328 \mathrm{bp}$ \\
\hline Flk1 & F: GGCCCAATAATCAGAGTGGCA; R: CCÁGTGTCATTTCCGATCACTTT & $109 \mathrm{bp}$ \\
\hline GFAP(1) & F: AGGTCCATGTGGAGCTTGAC; R: GCCATTGCCTCATACTGCGT & $82 \mathrm{bp}$ \\
\hline GFAP(2) & F: GTACCAGGACCTGCTCAAT; R: CAACTATCCTGCTTCTGCTC & $321 \mathrm{bp}$ \\
\hline Klf4 & $\begin{array}{l}\text { F: TGATTGTAGTGCTTTCTGGCTGGGCTCC } \\
\text { R: ACGATCGTGGCCCCGGAAAAGGACC }\end{array}$ & $397 \mathrm{bp}$ \\
\hline MAP2 & F: CGAAGCGCCAATGGATTCC; R: TGAACTATCCTTGCAGACACCT & \\
\hline nestin & F: CTGCTACCCTTGAGACACCTG; R: GGGCTCTGATCTCTGCATCTAC & $141 \mathrm{bp}$ \\
\hline NG2 & F: CTTTGACCCTGACTATGTTGGC; R: TGCAGGCGTCCAGAGTAGA & $202 \mathrm{bp}$ \\
\hline PDGFR $\beta$ & F: AGCACCTTCGTTCTGACCTG; R: TATTCTCCCGTGTCTAGCCCA & $152 \mathrm{bp}$ \\
\hline Slug & F: TGTGACAAGGAATATGTGAGCC; R: TGAGCCCTCAGATTTGACCTG & $203 \mathrm{bp}$ \\
\hline Sox 2 & F: TGGACAGTTACGCGCACAT; R: CGAGTAGGACATGCTGTAGGT & $215 \mathrm{bp}$ \\
\hline Sox9 & F: AGCGAACGCACATCAAGAC; R: CTGTAGGCGATCTGTTGGGG & $85 \mathrm{bp}$ \\
\hline Tuj1 & F: AGTGTGAAAACTGCGACTGC; R: ACGACGCTGAAGGTGTTCAT & $142 \mathrm{bp}$ \\
\hline
\end{tabular}


Table 2. Clinical Characteristics of Two Stroke Patients Treated by Decompressive Craniectomy and Partial Lobectomy for Diffuse Cerebral INFARCTION

\begin{tabular}{|c|c|c|c|c|c|}
\hline Patients & Age & Sex & $\begin{array}{c}\text { Time from } \\
\text { onset to } \\
\text { surgery }\end{array}$ & $\begin{array}{c}\text { Infarcted } \\
\text { territory }\end{array}$ & $\begin{array}{c}\text { Recanalization } \\
\text { therapy }\end{array}$ \\
\hline Case 1 & 79 & Female & $36 \mathrm{~h}$ & Cerebrum & - \\
\hline Case 2 & 81 & Female & $120 \mathrm{~h}$ & Cerebrum & + \\
\hline
\end{tabular}

showed extensive low-density areas in the right internal carotid artery territory (Fig. 1A), while diffusion-weighted MRI showed a large early hyperintensity in the same territory (Fig. 1B). She thus was not a candidate for reperfusion therapy. The day after admission, she exhibited symptoms of cerebral herniation, including respiratory arrest and anisocoria. She received decompressive craniotomy and partial lobectomy $36 \mathrm{~h}$ after ischemic onset (Fig. 1C). Postoperative CT showed that the surgery was successful (Fig. 1D).

The second case (Case 2, Table 2) was an 81-year-old female admitted to a local hospital where she received endovascular thrombectomy for right middle cerebral artery occlusion. Partial reperfusion (thrombolysis in cerebral infarction scale 2A) was obtained by endovascular thrombectomy. On day 4 after treatment, brain CT showed a large bilateral low-density area and right hemorrhagic transformation. She was transported to our hospital and received decompressive craniotomy and partial lobectomy 5 days after ischemic onset. Postoperative CT revealed that the surgery was successful (Supplementary Fig. S1A; Supplementary Data are available online at www.liebertpub .com/scd).

\section{Histological examination of poststroke brain tissue sections}

We first conducted histological analysis of poststroke brain tissue obtained from the first patient. H\&E staining revealed numerous pyknotic cell nuclei (Fig. 2A), suggesting widespread apoptosis/necrosis. To confirm this, brain sections were subjected to TUNEL, which revealed many apoptotic/necrotic cells in poststroke areas (Fig. 2B). Alternatively, mature neural cells, including neurons [Tuj $1^{+}$ (Fig. 2C) and $\mathrm{MAP2}^{+}$cells (Fig. 2D)] and astrocytes

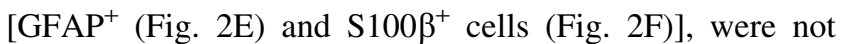
observed in these regions. These findings indicate that most mature neural cells had already undergone apoptosis/ necrosis and died by the time of tissue excision, consistent with histological findings in mice at the same time point following ischemia (Supplementary Fig. S2A-G).

In mice, we previously demonstrated that even under conditions of permanent ischemia in which mature neural cells did not survive, iSCs developed within postischemic areas $[12,13]$. Based on these findings, we examined whether iSCs were induced within the poststroke human brain tissue obtained from the first patient. To this end, we investigated the presence of nestin ${ }^{+}$cells, as we previously showed that mouse iSCs express the NSPC marker nestin $[12,13]$.

Consistent with these findings in mice, nestin ${ }^{+}$cells were observed within poststroke areas (Fig. 2G). Our previous rodent experiments also suggested that nestin ${ }^{+}$iSCs are likely derived from iPCs [12,13,22]; so we further investigated whether nestin ${ }^{+}$cells were localized near blood vessels. Indeed, nestin ${ }^{+}$cells were found near $\mathrm{vWF}^{+}$endothelial cells (ECs) (Fig. 2H) and coexpressed the pericytic markers aSMA (Fig. 2I-K) and NG2 (Fig. 2 L-N).

Similar histological findings were obtained for the poststroke brain tissue from the second patient (Supplementary Fig. S1B-H).

\section{Characterization of human iSCs likely derived from brain pericytes}

To further describe the properties of these nestin ${ }^{+}$cells, the poststroke brain tissue obtained from the first patient was dissociated by passage through needles to obtain single-cell suspensions. These dissociated cells were incubated under adherent culture conditions in a medium that promotes the proliferation of iSCs [12-14]. These adherent cells (Fig. 3A) were proliferative and expressed the nuclear marker of dividing cells Ki67 (Fig. 3B). Immunocytochemical analysis showed that these adherent cells expressed nestin (Fig. 3C) as well as the pericytic markers $\alpha$ SMA (Fig. 3D-F), NG2 (Fig. 3G-I), and PDGFR $\beta$ (Fig. 3J-L).

To examine the possibility that these nestin ${ }^{+}$cells are reactive astrocytes [6], adherent cells were coimmunostained with antibodies against nestin and GFAP. However, we found that no nestin ${ }^{+}$cells coexpressed GFAP (Fig. 3MO). Consistent with immunocytochemical findings, the GFAP
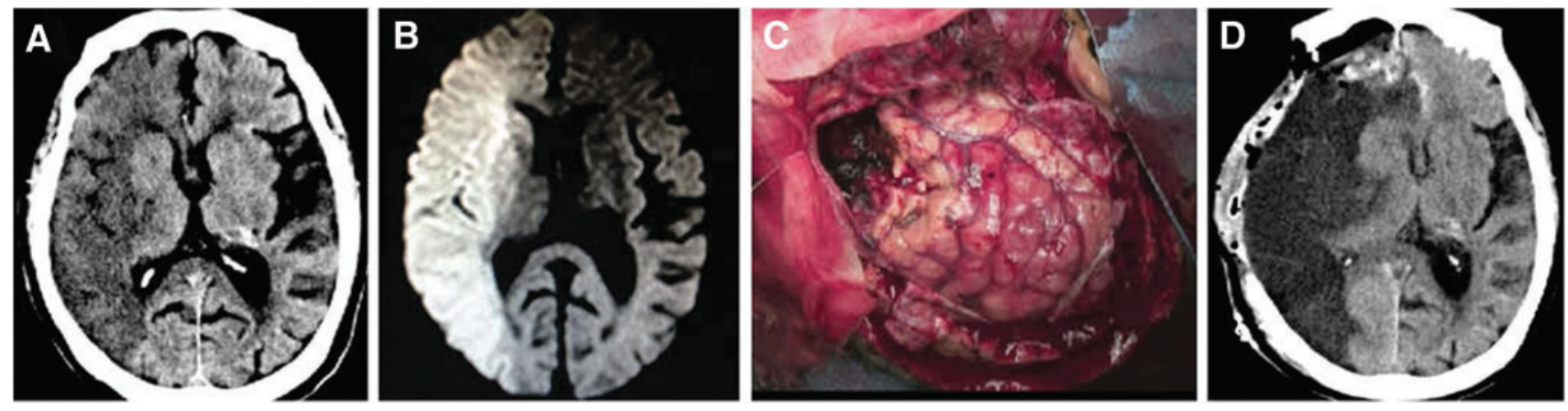

FIG. 1. Clinical history of the first patient with stroke. CT (A) and diffusion-weighted MRI (B) of a 79-year-old female stroke patient. Decompressive craniectomy and partial lobectomy were performed for stroke treatment (C). Postoperative CT following the surgery (D). CT, computed tomography; MRI, magnetic resonance imaging. 

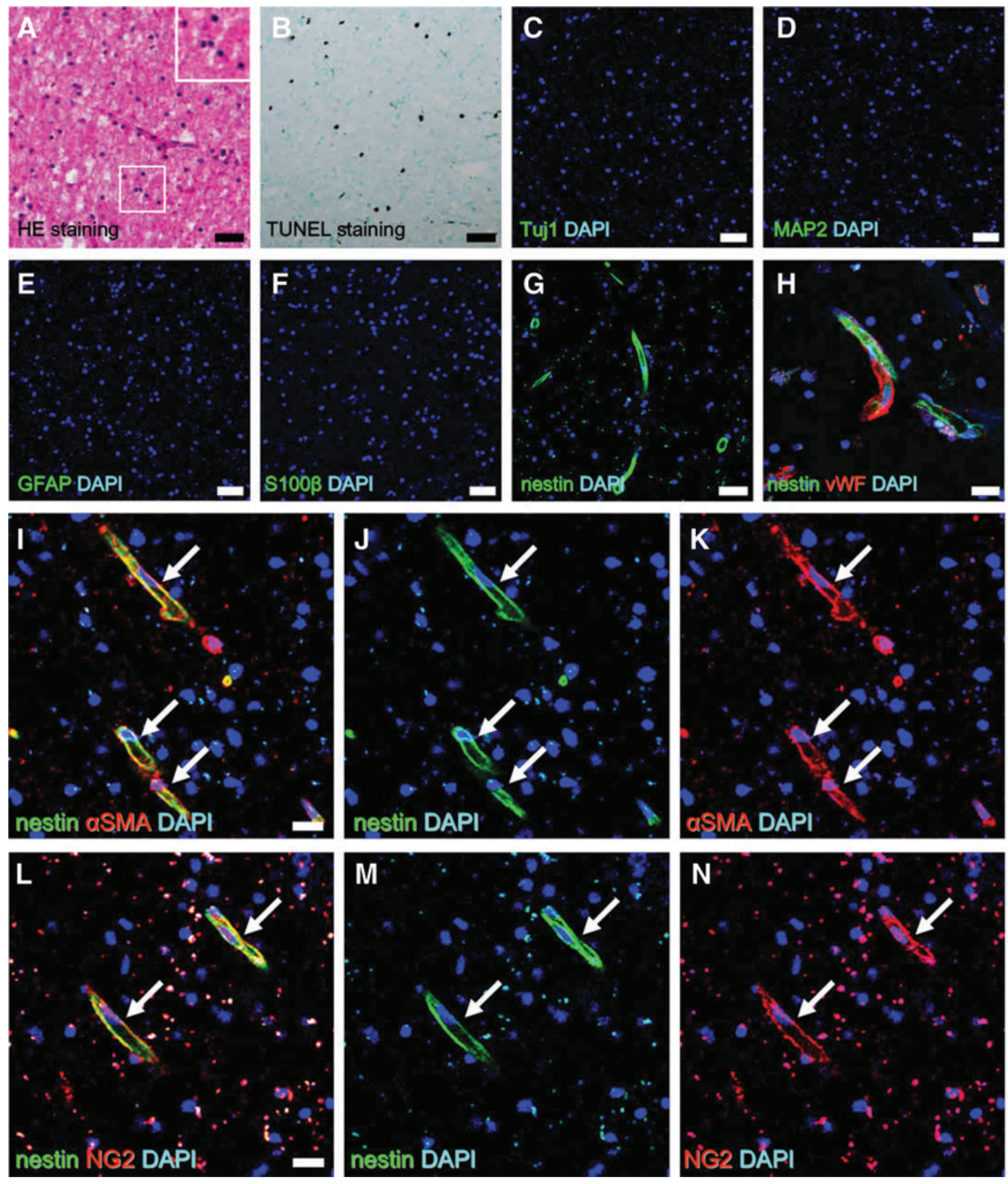

FIG. 2. Histological findings of poststroke human brain tissue sections obtained from the first patient. H\&E staining of surgically excised tissue revealed cells within the ischemic areas undergoing nuclear pyknotic changes (A). TUNEL staining showing many cells undergoing apoptosis/necrosis $(\mathbf{B})$. Immunohistochemistry showing the absence of healthy mature neural cells, such as neurons [Tuj1 (C: green) and DAPI (C: blue); MAP2 (D: green) and DAPI (D: blue)] and astrocytes [GFAP (E: green) and DAPI (E: blue); S100ß (F: green) and DAPI (F: blue)] within the ischemic area, while nestin ${ }^{+}$(stem like) cells were observed within these regions [nestin (G: green) and DAPI (G: blue)]. Nestin ${ }^{+}$cells were observed near vWF ${ }^{+}$ECs [nestin $(\mathbf{H}$ : green), vWF (H: red), and DAPI (H: blue)]. Nestin ${ }^{+}$cells expressed the pericytic markers $\alpha$ SMA [nestin (I, J: green), $\alpha$ SMA (I, K: red), and DAPI (I-K: blue)] (arrows) and NG2 [nestin (L, M: green), NG2 (L, N: red), and DAPI (L-N: blue)] (arrows). Scale bars $=50 \mu \mathrm{m}(\mathbf{A}-\mathbf{G})$ and $20 \mu \mathrm{m}(\mathbf{H}, \mathbf{I}, \mathbf{L}) . \alpha \mathrm{SMA}$, alpha smooth muscle actin; EC, endothelial cell; H\&E, hematoxylin and eosin.

mRNA expression was undetectable by RT-PCR analysis using two different primers, although GFAP mRNA expression was detected in hACs used as positive control (Fig. 3P). Thus, these nestin ${ }^{+}$cells are not reactive astrocytes.

Although nestin ${ }^{+}$cells expressed pericytic markers, it has been reported that nestin is also expressed in vascular lineage cells, including ECs [23,24]. To investigate whe- ther these putative iSCs contain EC populations, RT-PCR was performed. The result showed that these putative iSCs lacked expression of EC-related genes, such as $C D 31, C D H 5$, and $F l k 1$, although expression of all these genes was detected in $\mathrm{hECs}$ used as a positive control (Fig. 3Q).

Brain pericytes are derived not only from the mesoderm but also from the neural crest [25]; so we next examined 

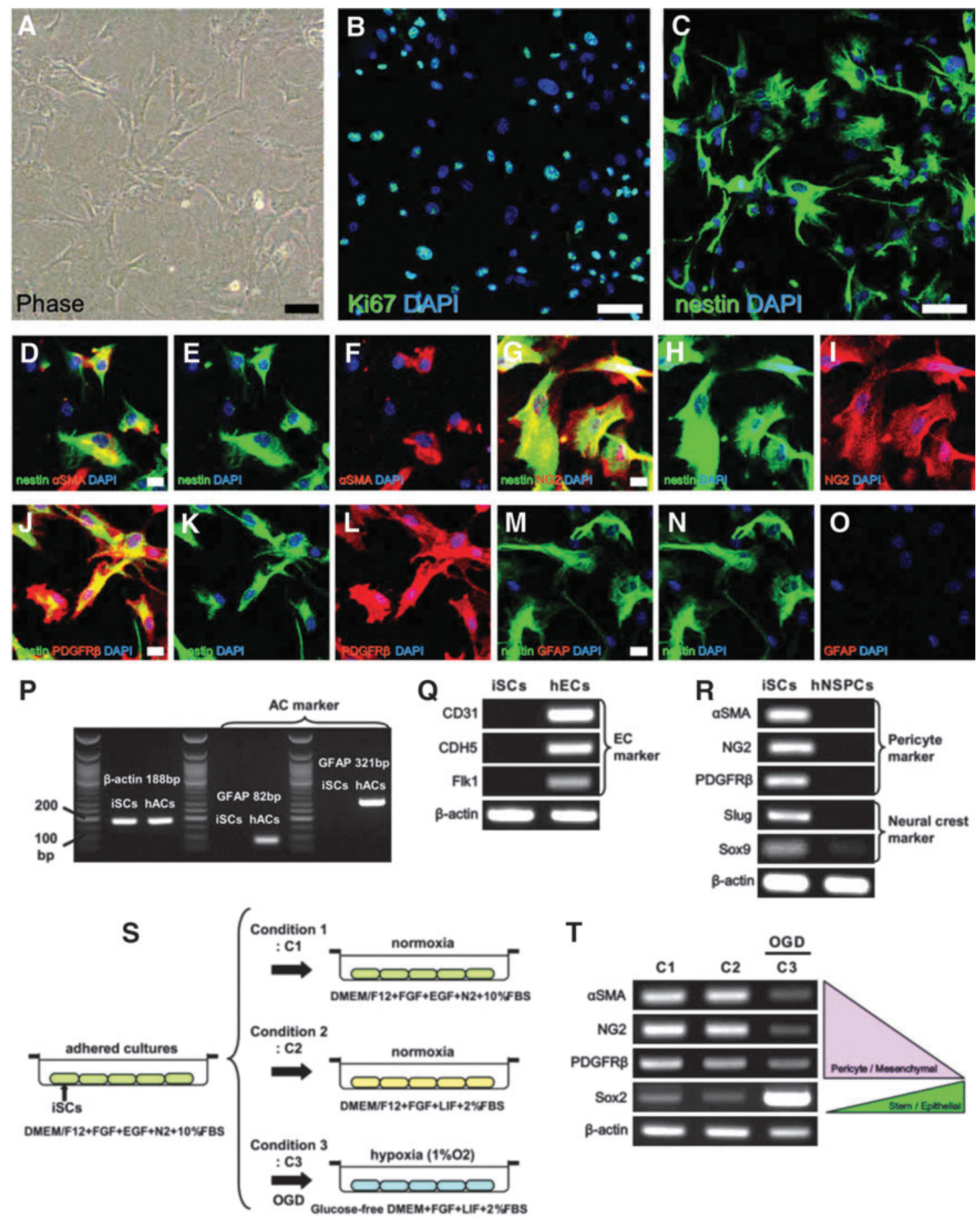

FIG. 3. Isolation and characterization of human iSCs obtained from the first patient. Putative iSCs (A) widely expressed the dividing cell marker Ki67 [Ki67 (B: green) and DAPI (B: blue)] and the neural stem cell marker nestin [nestin (C: green) and DAPI (C: blue)]. Immunohistochemical analysis revealed that these nestin ${ }^{+}$cells also expressed the pericytic markers $\alpha$ SMA [nestin (D, E: green), $\alpha$ SMA (D, F: red), and DAPI (D-F: blue)], NG2 [nestin (G, H: green), NG2 (G, I: red), and DAPI (G-I: blue)], and PDGFR $\beta$ [nestin ( $\mathbf{J}, \mathbf{K}:$ green), PDGFR $\beta$ (J, L: red), and DAPI (J-L: blue)], but not GFAP [nestin (M, N: green), GFAP (M, O: red), and DAPI (M-O: blue)]. PCR analysis indicated that putative iSCs did not express astrocytic (P) and endothelial lineage markers $(\mathbf{Q})$, but did express pericytic and neural crest lineage markers (R). Under OGD (S), these putative iSCs downregulated pericyte-related genes and upregulated stem/neuroepithelial cell genes, presumably through MET (T). Scale bars $=50 \mu \mathrm{m}(\mathbf{A}), 100 \mu \mathrm{m}(\mathbf{B}, \mathbf{C})$, and $20 \mu \mathrm{m}(\mathbf{D}, \mathbf{G}, \mathbf{J}, \mathbf{M})$. iSC, ischemiainduced multipotent stem cells; MET, mesenchymal-epithelial transition; OGD, oxygen-glucose deprivation. 
whether adherent nestin ${ }^{+}$cells express neural crest markers. Indeed, PCR analysis revealed expression not only of pericytic markers ( $\alpha S M A, N G 2$, and $P D G F R \beta)$ but also of neural crest markers (Slug and Sox9). In contrast, few neuroepithelium-derived hNSPCs used as control expressed these neural crest markers (Fig. 3R). These findings strongly suggest that human candidate iSCs are likely derived from brain pericytes, which are neural crest lineage derivatives, consistent with previous findings in the mouse $[12,13]$.

When subjected to OGD as a model of brain ischemia/ hypoxia (Fig. 3S) [13,20], these putative iSCs showed a decreased expression of pericytic markers (Fig. 3T), indicating loss of the original mesenchymal phenotype. Conversely, they exhibited an increased expression of the stem cell marker Sox2 (Fig. 3T), which is also a hallmark of neuroepithelial cells [26]. These results support our previous findings that mouse brain pericytes can develop stemness under ischemia/hypoxia through mesenchymal-epithelial transition (MET) [13,20].

Similar lineage characteristics were observed in putative iSCs obtained from the second patient (Supplementary Fig. S3A-Q).

\section{Human iSCs have multipotent stem cell activity}

Finally, we investigated whether these human candidate iSCs have a multipotent stem cell activity. Pericytes are reported to share certain traits with MSCs [27-31; so we first investigated whether adherent cells obtained from the first patient express the MSC markers CD44, CD90, CD105, and CD166. Indeed, subpopulations expressed CD44 (72.8\%), CD90 (53.7\%), CD105 (90.3\%), and CD166 (75.3\%), but not CD19 or CD45 (Fig. 4A). Similarly, hMSCs used as a positive control expressed CD44 (99.7\%), CD90 (99.0\%), CD105 (99.8\%), and CD166 (99.5\%), but not CD19 or CD45 (Fig. 4A). These findings show that human candidate iSCs and MSCs have similar cell surface marker patterns.

The neural crest contains multipotent stem cells with features of both neural and mesenchymal cells [32-35]; so we further compared the traits of adherent nestin ${ }^{+}$cells to hNSPCs and hMSCs, neural and mesenchymal lineage stem cells, respectively.

Although the NSPC marker nestin was observed in both putative iSCs and hNSPCs, hMSCs rarely expressed nestin. We next investigated whether putative iSCs expressed other stem and undifferentiated cell markers such as Sox2, c-myc, and Klf4. Sox 2 was expressed in all types of stem cells and was highly expressed in neuroepithelial cell-derived hNSPCs. However, $c$-myc and Klf4 were not observed in hNSPCs, although both markers were expressed in putative iSCs and hMSCs. Thus, putative human iSCs expressed various stem and undifferentiated cell markers, including nestin, Sox2, c-myc, and Klf4 (Fig. 4B), consistent with findings in the mouse $[12,13]$.

These human iSC-like cells also exhibited a high proliferation rate as evidenced by a doubling time of $66.7 \pm 5.1 \mathrm{~h}$ (Fig. 4C), but they maintained expression of stem cell markers (Fig. 4D), strongly suggesting multipotency. To confirm this, adherent putative iSCs were subjected to incubation under osteoblastic, adipogenic, and chondrocytic differentiation conditions. Under these respective conditions, they differentiated into osteocalcin ${ }^{+}$osteoblasts (Fig. 4E),
$\mathrm{FABP}^{+}$(Fig. 4F) and Oil red ${ }^{+}$adipocytes (Fig. 4G), and aggrecan $^{+}$chondrocytes (Fig. $4 \mathrm{H}$ ).

To investigate whether these multipotent adherent cells can also differentiate along neuronal lineage pathways, harvested cells were maintained in floating cultures under conditions that promote the formation of neurosphere-like cell clusters (Fig. 4I) [12-14,18]. iSCs formed cell clusters (Fig. 4J) and expressed the neuronal markers Tuj1 and MAP2 (Fig. 4K). Immunocytochemistry also showed that they differentiated into Tuj $1^{+}$neuronal cells (Fig. 4L). Similar multipotent stem cell activities were confirmed in putative iSCs obtained from the second patient (Supplementary Fig. S4A-I). Considered in aggregate, these results indicate that the adherent nestin ${ }^{+}$cells derived from two human stroke patients are iSCs similar to those described in mice.

\section{Discussion}

This is the first clinical report showing that iSCs are present within the poststroke human brain. In this study, we found that iSCs with neuronal differentiation potential were present within both the poststroke brain of a patient with permanent ischemia (Case 1) and a patient after ischemia/ reperfusion (Case 2). These findings are consistent with results in mouse showing that iSCs can be isolated from poststroke areas after ischemia/reperfusion injury [22] as well as after permanent ischemic injury [12-14,18]. In addition, we show that iSCs are likely derived from brain pericytes localized near blood vessels, consistent with findings in the mouse [12,13,22].

It is well documented that pericytes have the potential to function as multipotent stem cells [19,36-43]. However, our previous study of mice at different developmental stages, including embryonic, postnatal, and adult, revealed that stemness of brain pericytes gradually decreased during the postnatal period and was eventually lost in adulthood [44]. This indicates that brain pericytes exist as somatic cells rather than stem cells in the adult brain, although they are likely to retain the traits of stem cells up to the early postnatal period.

Although brain pericytes in adult mice did not have a stem cell activity under normal conditions, we found that they reacquired stemness in response to ischemia, presumably through cellular reprogramming by MET $[13,20]$. In support of this notion, this study showed that iSCs can be isolated from postischemic regions even in an aged human brain and iSCs further develop stemness through MET under ischemic/hypoxic conditions.

Ethical limitations preclude investigation of whether iSCs can also be isolated from healthy adult human brain tissue. However, our previous studies showed that iSCs only developed within poststroke areas, and no iSCs were isolated from nonischemic areas of the adult mouse brain [12,13,20]. This strongly suggests that iSCs are restricted to postischemic tissue in the adult human brain as well.

Although we do not know if similar multipotent stem cell populations are present in the ischemic tissue of organs other than the CNS (eg, postischemic heart following myocardial infarction), a recent study showed that skeletal muscle cells within injured regions acquired pluripotency through reprogramming, while skeletal muscle cells within the noninjured regions did not [45]. Therefore, although the precise mechanism by which stem cell populations are 

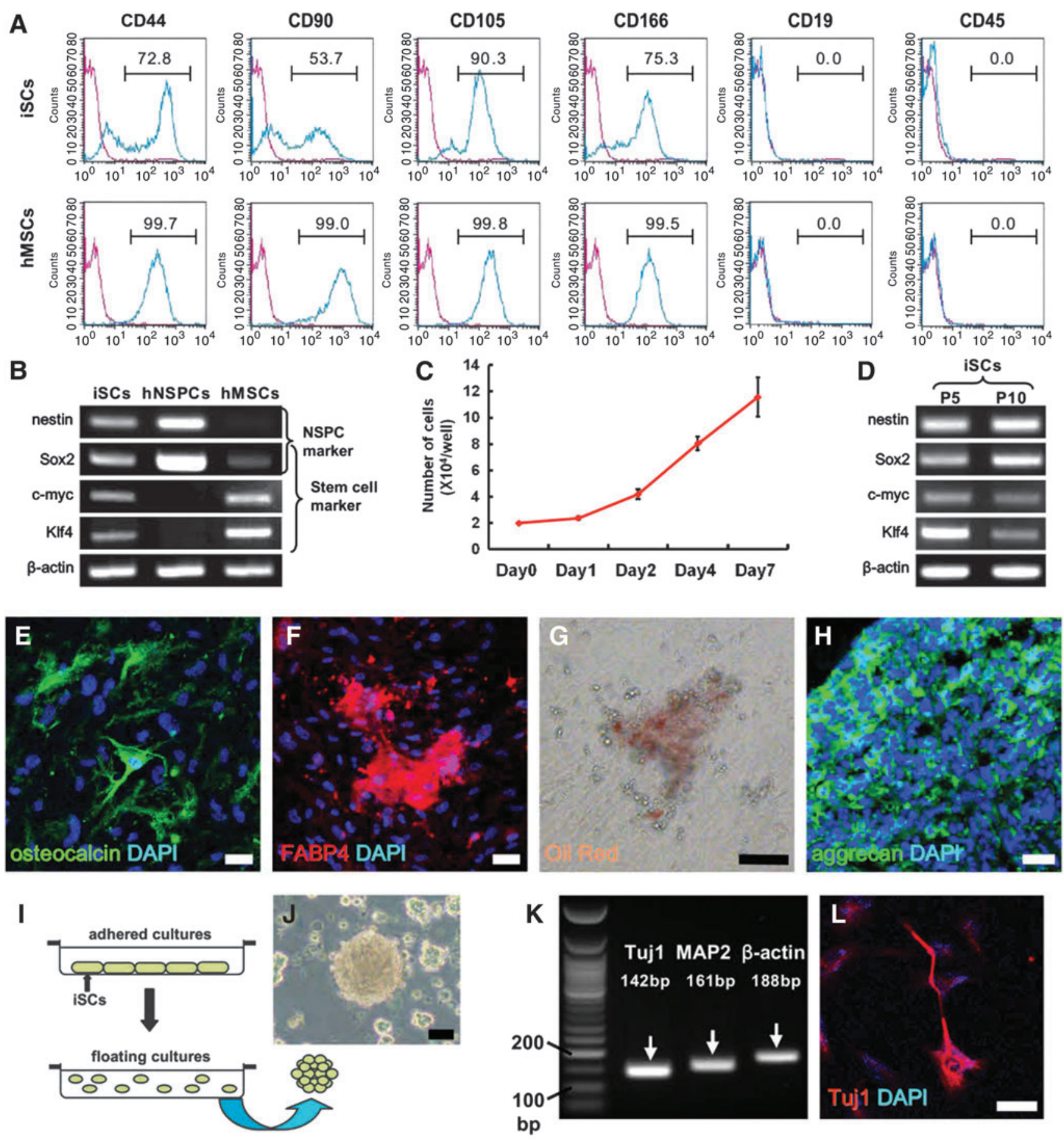

FIG. 4. Multipotent stem cell activity of human iSCs obtained from the first patient. Flow cytometric analysis demonstrated that putative human iSCs expressed multiple MSC markers (A). PCR analysis revealed expression of various marker genes for stem and undifferentiated cells (B). Putative iSCs displayed a high proliferative potential (C). PCR analysis revealed that iSCs maintained expression of stem cell markers even after several passages (P5, passage 5; P10, passage 10) (D). iSCs differentiated into osteocalcin ${ }^{+}$osteoblasts [osteocalcin (E: green) and DAPI (E: blue)] as well as FABP4 ${ }^{+}$ [FABP4 (F: red) and DAPI (F: blue)], and Oil Red $^{+}$adipocytes $(\mathbf{G})$ and aggrecan ${ }^{+}$chondrocytes [aggrecan $(\mathbf{H}:$ green) and DAPI (H: blue)]. iSCs that formed neurosphere-like cell clusters in suspended culture (I, J) expressed neuronal genes (K). Immunohistochemical analysis showed that the cell clusters differentiated into Tuj1 ${ }^{+}$neuronal cells [Tuj1 (L: red) and DAPI (L: red)]. Scale bars $=50 \mu \mathrm{m}(\mathbf{E}-\mathbf{H}, \mathbf{J}, \mathbf{L})$. MSC, mesenchymal stem cell. 
induced in response to ischemia/injury remains unclear, it is possible that multipotent stem cells are present in the damaged tissue of various organs following insult.

Multipotent pericytes differentiate not only into neuroectoderm (eg, neural cells) but also into mesoderm lineages (eg, adipocytes, osteoblasts, chondrocytes, and vascular cells) [19,36-43]. In this study, we found that human iSCs likely originating from iPCs differentiated into neural cells, adipocytes, or osteoblasts under appropriate conditions, consistent with the traits of rodent iSCs $[13,20]$.

It remains unclear if iPCs/iSCs contain subpopulations with a higher potential toward specific lineages (neurons, glia, vascular cells, etc.). If so, clinical applications could be greatly facilitated by their isolation. Birbrair and colleagues showed that multipotent pericytes in skeletal muscle are of two subtypes: nestin ${ }^{+} / \mathrm{NG} 2^{+}$type- 2 pericytes that differentiate into neural and vascular lineages and nestin ${ }^{-} / \mathrm{NG}^{+}$type- 1 pericytes that form adipogenic progenitors [19,38,39,43]. Furthermore, they showed that nestin ${ }^{+} / \mathrm{NG}^{+}$type- 2 pericytes have traits similar to neural progenitors with properties of NG2-glia [46]. The complex lineage relationships among pericytes, neural progenitors, and glia [27] must be elucidated for the selective lineage specifications required to regenerate functional neural tissue.

In this study, we showed that iSCs express similar phenotypic markers as MSCs. In addition, we demonstrated that iSCs have a multidifferentiation potential, also consistent with MSCs [31,47]. It is well-documented that MSCs localize within a perivascular niche in multiple organs, including bone marrow $[31,47]$, and a recent study reported that the adult brain also harbors multipotent MSCs in perivascular regions [48]. It remains unclear whether iSCs and perivascular brain MSCs are identical stem cells. However, it is believed that certain pericytes, in particular those derived from the mesoderm, can produce MSCs [28-30]. Thus, it may be natural that iSCs, which are likely pericyte derivatives, have traits similar to MSCs.

Although the precise origin of brain pericytes remains unclear, it is reported that forebrain pericytes originate from the neural crest, while pericytes in other brain regions are mesoderm derivatives [25]. In support of this notion, this study showed that human iSCs isolated from frontotemporal regions expressed markers of both the neural crest and pericytes. The neural crest contains multipotent stem cells that possess features of both neural and mesenchymal cells [32-35], and this study showed that iSCs express markers of NSPCs, such as nestin and Sox2, in addition to MSC markers. This may explain why iSCs can differentiate into neural lineages as well as nonneural lineages.

Many recent studies have investigated stem cell-based therapies using ectopic multipotent stem cells, such as MSCs, adipose-derived stem cells, and hematopoietic stem cells for various CNS disorders, including ischemic stroke $[18,49,50]$. This study strongly suggests that iSCs, which share several traits with MSCs, are locally induced in the perivascular regions of the poststroke human brain. Cell-based therapies based on regionally induced multipotent iSCs may be a superior strategy for CNS regeneration following injury, since several studies found that exogenously administered stem cells became trapped inside the lungs following intravenous transplantation and only a small fraction accumulated in injured areas [18,51].
In addition, our previous studies showed that mouse iSCs can contribute to neurogenesis with appropriate support $[14,18]$, although they largely underwent cell death without it $[52,53]$. This indicates that administration of factors that regulate iSC fate (eg, cell proliferation, differentiation, survival, and migration) could become novel therapies for stroke patients. In this study, we found that human iSCs can rapidly expand in vitro, while maintaining stemness. These findings indicate that isolated human iSCs may be used as autologous stem cells for transplantation in patients following severe stroke.

Many outstanding questions and issues remain to be addressed before iSC-based therapies are feasible. For instance, the optimal period for iSC isolation is unknown. In addition, we need to identify specific factors that can regulate the proliferation, differentiation, and migration of iSCs. We also need to assess whether the phenotypes of iSCs differ depending on the brain region (forebrain vs. other regions). Furthermore, excessive proliferation of iSCs may cause unexpected vascular diseases, such as lipohyalinosis and calcification, in the long term after stroke because our iSCs differentiated into adipocytes and osteoblasts in addition to neuronal lineages. There are still many unknowns regarding human iSCs, but our findings provide the basis for further investigations on a novel iSC-based therapeutic strategy for patients with stroke.

In conclusion, we demonstrated that iSCs are present within the poststroke human brain. Ischemia-induced multipotent stem cells can differentiate into neuronal cells and, thus, could have an important role in CNS repair following ischemic stroke. A more complete understanding of iSC properties and methods for transplantation and/or in vivo regulation could make neural regeneration a reality for stroke patients.

\section{Acknowledgments}

This work was partially supported by Japan Society for the Promotion of Science (JSPS) KAKENHI (15K06723), a Strategic Program Grant for Research Institute Development in Private Institute from Ministry of Education, Culture, Sports, Science and Technology (MEXT) in Japan, and Japan Agency for Medical Research and Development (AMED).

\section{Author Disclosure Statement}

The authors declare no conflict of interests.

\section{References}

1. Mokin M, T Kass-Hout, O Kass-Hout, TM Dumont, P Kan, KV Snyder, LN Hopkins, AH Siddiqui and EI Levy. (2012). Intravenous thrombolysis and endovascular therapy for acute ischemic stroke with internal carotid artery occlusion: a systematic review of clinical outcomes. Stroke 43:2362-2368.

2. Zaidat OO, JI Suarez, C Santillan, JL Sunshine, RW Tarr, VH Paras, WR Selman and DM Landis. (2002). Response to intra-arterial and combined intravenous and intra-arterial thrombolytic therapy in patients with distal internal carotid artery occlusion. Stroke 33:1821-1826.

3. Jackson EL, JM Garcia-Verdugo, S Gil-Perotin, M Roy, A Quinones-Hinojosa, S VandenBerg and A Alvarez-Buylla. 
(2006). PDGFR alpha-positive B cells are neural stem cells in the adult SVZ that form glioma-like growths in response to increased PDGF signaling. Neuron 51:187-199.

4. Doetsch F, I Caille, DA Lim, JM Garcia-Verdugo and A Alvarez-Buylla. (1999). Subventricular zone astrocytes are neural stem cells in the adult mammalian brain. Cell 97: 703-716.

5. Kondo T and M Raff. (2000). Oligodendrocyte precursor cells reprogrammed to become multipotential CNS stem cells. Science 289:1754-1757.

6. Shimada IS, BM Peterson and JL Spees. (2010). Isolation of locally derived stem/progenitor cells from the periinfarct area that do not migrate from the lateral ventricle after cortical stroke. Stroke 41:e552-e560.

7. Moreno-Manzano V, FJ Rodriguez-Jimenez, M GarciaRosello, S Lainez, S Erceg, MT Calvo, M Ronaghi, M Lloret, R Planells-Cases, JM Sanchez-Puelles and M Stojkovic. (2009). Activated spinal cord ependymal stem cells rescue neurological function. Stem Cells 27:733-743.

8. Arsenijevic Y, JG Villemure, JF Brunet, JJ Bloch, N Deglon, C Kostic, A Zurn and P Aebischer. (2001). Isolation of multipotent neural precursors residing in the cortex of the adult human brain. Exp Neurol 170:48-62.

9. Kallur T, V Darsalia, O Lindvall and Z Kokaia. (2006). Human fetal cortical and striatal neural stem cells generate region-specific neurons in vitro and differentiate extensively to neurons after intrastriatal transplantation in neonatal rats. J Neurosci Res 84:1630-1644.

10. Joo KM, BG Kang, JY Yeon, YJ Cho, JY An, HS Song, JH Won, SJ Kim, SC Hong and DH Nam. (2013). Experimental and clinical factors influencing long-term stable in vitro expansion of multipotent neural cells from human adult temporal lobes. Exp Neurol 240:168-177.

11. Yokoyama A, A Sakamoto, K Kameda, Y Imai and J Tanaka. (2006). NG2 proteoglycan-expressing microglia as multipotent neural progenitors in normal and pathologic brains. Glia 53:754-768.

12. Nakagomi T, Z Molnar, A Nakano-Doi, A Taguchi, O Saino, S Kubo, M Clausen, H Yoshikawa, N Nakagomi and T Matsuyama. (2011). Ischemia-induced neural stem/ progenitor cells in the pia mater following cortical infarction. Stem Cells Dev 20:2037-2051.

13. Nakagomi T, S Kubo, A Nakano-Doi, R Sakuma, S Lu, A Narita, M Kawahara, A Taguchi and T Matsuyama. (2015). Brain vascular pericytes following ischemia have multipotential stem cell activity to differntiate into neural and vascular lineage cells. Stem Cells 33:1962-1974.

14. Nakagomi N, T Nakagomi, S Kubo, A Nakano-Doi, O Saino, M Takata, H Yoshikawa, DM Stern, T Matsuyama and A Taguchi. (2009). Endothelial cells support survival, proliferation, and neuronal differentiation of transplanted adult ischemia-induced neural stem/progenitor cells after cerebral infarction. Stem Cells 27:2185-2195.

15. Nakayama D, T Matsuyama, H Ishibashi-Ueda, T Nakagomi, Y Kasahara, H Hirose, A Kikuchi-Taura, DM Stern, H Mori and A Taguchi. (2010). Injury-induced neural stem/ progenitor cells in post-stroke human cerebral cortex. Eur J Neurosci 31:90-98.

16. Nakagomi T, O Kitada, K Kuribayashi, H Yoshikawa, K Ozawa, S Ogawa and T Matsuyama. (2004). The 150-kilodalton oxygen-regulated protein ameliorates lipopolysaccharideinduced acute lung injury in mice. Am J Pathol 165:12791288 .
17. Nakagomi T, A Taguchi, Y Fujimori, O Saino, A NakanoDoi, S Kubo, A Gotoh, T Soma, H Yoshikawa, et al. (2009). Isolation and characterization of neural stem/progenitor cells from post-stroke cerebral cortex in mice. Eur J Neurosci 29:1842-1852.

18. Nakano-Doi A, T Nakagomi, M Fujikawa, N Nakagomi, S Kubo, S Lu, H Yoshikawa, T Soma, A Taguchi and $\mathrm{T}$ Matsuyama. (2010). Bone marrow mononuclear cells promote proliferation of endogenous neural stem cells through vascular niches after cerebral infarction. Stem Cells 28: 1292-1302.

19. Birbrair A, T Zhang, ZM Wang, ML Messi, GN Enikolopov, A Mintz and O Delbono. (2013). Role of pericytes in skeletal muscle regeneration and fat accumulation. Stem Cells Dev 22:2298-2314.

20. Sakuma R, M Kawahara, A Nakano-Doi, A Takahashi, Y Tanaka, A Narita, S Kuwahara-Otani, T Hayakawa, H Yagi, T Matsuyama and T Nakagomi. (2016). Brain pericytes serve as microglia-generating multipotent vascular stem cells following ischemic stroke. J Neuroinflammation 13:57.

21. Wystrychowski W, B Patlolla, Y Zhuge, E Neofytou, RC Robbins and RE Beygui. (2016). Multipotency and cardiomyogenic potential of human adipose-derived stem cells from epicardium, pericardium, and omentum. Stem Cell Res Ther 7:84.

22. Nakata M, T Nakagomi, M Maeda, A Nakano-Doi, Y Momota and T Matsuyama. (2017). Induction of Perivascular Neural Stem Cells and Possible Contribution to Neurogenesis Following Transient Brain Ischemia/Reperfusion Injury. Transl Stroke Res 8:131-143.

23. Mokry J, D Cizkova, S Filip, J Ehrmann, J Osterreicher, Z Kolar and D English. (2004). Nestin expression by newly formed human blood vessels. Stem Cells Dev 13:658-664.

24. Suzuki S, J Namiki, S Shibata, Y Mastuzaki and H Okano. (2010). The neural stem/progenitor cell marker nestin is expressed in proliferative endothelial cells, but not in mature vasculature. J Histochem Cytochem 58:721-730.

25. Etchevers HC, C Vincent, NM Le Douarin and GF Couly. (2001). The cephalic neural crest provides pericytes and smooth muscle cells to all blood vessels of the face and forebrain. Development 128:1059-1068.

26. Cimadamore F, K Fishwick, E Giusto, K Gnedeva, G Cattarossi, A Miller, S Pluchino, LM Brill, M BronnerFraser and AV Terskikh. (2011). Human ESC-derived neural crest model reveals a key role for SOX2 in sensory neurogenesis. Cell Stem Cell 8:538-551.

27. Nakagomi $T, A$ Nakano-Doi, $M$ Kawamura and $T$ Matsuyama. (2015). Do vascular pericytes contribute to neurovasculogenesis in the central nervous system as multipotent vascular stem cells? Stem Cells Dev 24:17301739.

28. Feng J, A Mantesso, C De Bari, A Nishiyama and PT Sharpe. (2011). Dual origin of mesenchymal stem cells contributing to organ growth and repair. Proc Natl Acad Sci U S A 108:6503-6508.

29. Caplan AI. (2008). All MSCs are pericytes? Cell Stem Cell 3:229-230.

30. Corselli M, CW Chen, M Crisan, L Lazzari and B Peault. (2010). Perivascular ancestors of adult multipotent stem cells. Arterioscler Thromb Vasc Biol 30:1104-1109.

31. Crisan M, S Yap, L Casteilla, CW Chen, M Corselli, TS Park, G Andriolo, B Sun, B Zheng, et al. (2008). A peri- 
vascular origin for mesenchymal stem cells in multiple human organs. Cell Stem Cell 3:301-313.

32. Calloni GW, C Glavieux-Pardanaud, NM Le Douarin and E Dupin. (2007). Sonic Hedgehog promotes the development of multipotent neural crest progenitors endowed with both mesenchymal and neural potentials. Proc Natl Acad Sci U S A 104:19879-19884.

33. Calloni GW, NM Le Douarin and E Dupin. (2009). High frequency of cephalic neural crest cells shows coexistence of neurogenic, melanogenic, and osteogenic differentiation capacities. Proc Natl Acad Sci U S A 106: 8947-8952.

34. Wislet-Gendebien S, E Laudet, V Neirinckx, P Alix, P Leprince, A Glejzer, C Poulet, B Hennuy, L Sommer, O Shakhova and B Rogister. (2012). Mesenchymal stem cells and neural crest stem cells from adult bone marrow: characterization of their surprising similarities and differences. Cell Mol Life Sci 69:2593-2608.

35. Isern J, A Garcia-Garcia, AM Martin, L Arranz, D MartinPerez, C Torroja, F Sanchez-Cabo and S Mendez-Ferrer. (2014). The neural crest is a source of mesenchymal stem cells with specialized hematopoietic stem cell niche function. Elife 3:e03696.

36. Dore-Duffy P, A Katychev, X Wang and E Van Buren. (2006). CNS microvascular pericytes exhibit multipotential stem cell activity. J Cereb Blood Flow Metab 26:613-624.

37. Kabara M, J Kawabe, M Matsuki, Y Hira, A Minoshima, K Shimamura, A Yamauchi, T Aonuma, M Nishimura, et al. (2014). Immortalized multipotent pericytes derived from the vasa vasorum in the injured vasculature. A cellular tool for studies of vascular remodeling and regeneration. Lab Invest 94:1340-1354.

38. Birbrair A, T Zhang, ZM Wang, ML Messi, GN Enikolopov, A Mintz and O Delbono. (2012). Skeletal muscle pericyte subtypes differ in their differentiation potential. Stem Cell Res 10:67-84.

39. Birbrair A, T Zhang, ZM Wang, ML Messi, A Mintz and O Delbono. (2014). Pericytes: multitasking cells in the regeneration of injured, diseased, and aged skeletal muscle. Front Aging Neurosci 6:245.

40. Farrington-Rock C, NJ Crofts, MJ Doherty, BA Ashton, C Griffin-Jones and AE Canfield. (2004). Chondrogenic and adipogenic potential of microvascular pericytes. Circulation 110:2226-2232.

41. Dar A, H Domev, O Ben-Yosef, M Tzukerman, N ZeeviLevin, A Novak, I Germanguz, M Amit and J ItskovitzEldor. (2012). Multipotent vasculogenic pericytes from human pluripotent stem cells promote recovery of murine ischemic limb. Circulation 125:87-99.

42. Doherty MJ, BA Ashton, S Walsh, JN Beresford, ME Grant and AE Canfield. (1998). Vascular pericytes express osteogenic potential in vitro and in vivo. $\mathrm{J}$ Bone Miner Res 13:828-838.

43. Birbrair A, T Zhang, ZM Wang, ML Messi, JD Olson, A Mintz and O Delbono. (2014). Type-2 pericytes participate in normal and tumoral angiogenesis. Am J Physiol Cell Physiol 307:C25-C38.

44. Nakano-Doi A, T Nakagomi, R Sakuma, A Takahashi, Y Tanaka, M Kawamura and T Matsuyama. (2016). Expression patterns and phenotypic changes regarding stemness in brain pericytes in health and disease. J Stem Cell Res Ther 6.

45. Vojnits K, H Pan, X Mu and Y Li. (2015). Characterization of an injury induced population of muscle-derived stem cell-like cells. Sci Rep 5:17355.
46. Birbrair A, T Zhang, ZM Wang, ML Messi, GN Enikolopov, A Mintz and O Delbono. (2013). Skeletal muscle neural progenitor cells exhibit properties of NG2-glia. Exp Cell Res 319:45-63.

47. Tropel P, D Noel, N Platet, P Legrand, AL Benabid and F Berger. (2004). Isolation and characterisation of mesenchymal stem cells from adult mouse bone marrow. Exp Cell Res 295:395-406.

48. Paul G, I Ozen, NS Christophersen, T Reinbothe, J Bengzon, E Visse, $\mathrm{K}$ Jansson, $\mathrm{K}$ Dannaeus, $\mathrm{C}$ HenriquesOliveira, et al. (2012). The adult human brain harbors multipotent perivascular mesenchymal stem cells. PLoS One 7:e35577.

49. Honma T, O Honmou, S Iihoshi, K Harada, K Houkin, H Hamada and JD Kocsis. (2006). Intravenous infusion of immortalized human mesenchymal stem cells protects against injury in a cerebral ischemia model in adult rat. Exp Neurol 199:56-66.

50. Zhou F, S Gao, L Wang, C Sun, L Chen, P Yuan, H Zhao, Y Yi, Y Qin, et al. (2015). Human adipose-derived stem cells partially rescue the stroke syndromes by promoting spatial learning and memory in mouse middle cerebral artery occlusion model. Stem Cell Res Ther 6:92.

51. Fischer UM, MT Harting, F Jimenez, WO MonzonPosadas, H Xue, SI Savitz, GA Laine and CS Cox, Jr. (2009). Pulmonary passage is a major obstacle for intravenous stem cell delivery: the pulmonary first-pass effect. Stem Cells Dev 18:683-692.

52. Saino O, A Taguchi, T Nakagomi, A Nakano-Doi, S Kashiwamura, N Doe, N Nakagomi, T Soma, H Yoshikawa, et al. (2010). Immunodeficiency reduces neural stem/progenitor cell apoptosis and enhances neurogenesis in the cerebral cortex after stroke. J Neurosci Res 88:2385-2397.

53. Takata M, T Nakagomi, S Kashiwamura, A Nakano-Doi, O Saino, N Nakagomi, H Okamura, O Mimura, A Taguchi and T Matsuyama. (2012). Glucocorticoid-induced TNF receptor-triggered $\mathrm{T}$ cells are key modulators for survival/ death of neural stem/progenitor cells induced by ischemic stroke. Cell Death Differ 19:756-767.

Address correspondence to: Takayuki Nakagomi, MD, PhD Institute for Advanced Medical Sciences Hyogo College of Medicine 1-1 Mukogawacho Nishinomiya 663-8501 Japan

E-mail: nakagomi@hyo-med.ac.jp

Shinichi Yoshimura, MD, PhD Department of Neurosurgery Hyogo College of Medicine 1-1 Mukogawacho Nishinomiya 663-8501 Japan

E-mail: shinyoshi523@gmail.com

Received for publication November 23, 2016 Accepted after revision March 21, 2017

Prepublished on Liebert Instant Online March 21, 2017 